\title{
The Galactic Archaeology with HERMES Survey
}

\author{
G. M. De Silva ${ }^{1}$
}

Australian Astronomical Observatory

105, Delhi Rd, North Ryde, NSW 2113, Australia.

E-mail: gayandhi.desilvalaao.gov.au

\section{K. C. Freeman}

Research School of Astronomy \& Astrophysics, Australian National University

Mt Stromlo Observatory, Cotter Rd, Weston, ACT 2611, Australia

\section{J. Bland-Hawthorn}

Institute of Astronomy, School of Physics

University of Sydney, Camperdown, NSW 2006, Australia

\section{B. Zucker}

Macquarie University Research Centre in Astronomy, Astrophysics \& Astrophotonics

Department of Physics \& Astronomy, Macquarie University, NSW 2109 Australia

The goal of Galactic Archaeology is to reconstruct the lost stellar substructures of the protogalaxy, thereby obtaining a detailed physical picture of the formation and evolution of the Galaxy. The Galactic Archaeology with HERMES (GALAH) Survey is a major Australian-led project to commence in 2013 using the Anglo-Australian Telescope of the Australian Astronomical Observatory (AAO). It will use the new state-of-the-art HERMES instrument to obtain high resolution spectra of over a million Galactic disk stars. HERMES provides $\sim 400$ multi-object capability and simultaneous spectra in four wavelength bands tailored to obtaining a range of chemical element lines, from light elements up to heavy neutron-capture elements. The GALAH survey will target stars down to $\mathrm{V} \sim 14 \mathrm{mag}$, and provide elemental abundances of well over 15 elements as well as accurate radial velocities.

XII International Symposium on Nuclei in the Cosmos

August 5-12, 2012

Cairns, Australia

$1 \quad$ Speaker




\section{Introduction}

The aim of Galactic Archaeology is to reconstruct the lost stellar substructures of the proto-galaxy, thereby obtaining a detailed physical picture of the formation and evolution of the Galaxy. The Galactic Archaeology with HERMES (GALAH) Survey is a major Australian-led project to commence in 2013 using the 3.9m Anglo-Australian Telescope of the Australian Astronomical Observatory (AAO). It will use the upcoming state-of-the-art HERMES instrument to obtain high resolution spectra of over a million Galactic disk stars, with the aim to chemically tag disk stars to common formation sites (Freeman \& Bland-Hawthorn 2002).

\section{The HERMES Instrument}

The High Efficiency and Resolution Multi-Element spectrograph (HERMES) is the next major instrument for the 3.9m Anglo-Australian Telescope (AAT), currently under construction at the Australian Astronomical Observatory (Barden et al., 2010). It will provide a unique and powerful new facility for high resolution multi-object spectroscopy. HERMES is expected to be available for science use in mid 2013.

HERMES provides simultaneous spectra in four wavelength bands tailored to obtaining a range of chemical element lines, from light elements up to heavy neutron-capture elements. It will provide a nominal spectral resolution of $\sim 28,000$, and a higher resolution mode of $\sim 48,000$. Together with the existing fiber positioning facility $(2 \mathrm{dF})$, the system is capable of observing up to 392 stars over a two-degree field of view in a single exposure in all four wavelength channels. The system will have a high efficiency, where the expected performance is to acheive a signal-to-noise ratio of 100 per resolution element for a $\mathrm{V}=14$ star within a total 1 hour exposure time.

\begin{tabular}{|c|c|c|c|c|}
\hline Channel & BLUE & GREEN & RED & IR \\
\hline Wavelength (nm) & $471.8-489.3$ & $564.9-587.3$ & $648.1-674.9$ & $759.0-789.0$ \\
\hline
\end{tabular}

Table 1: The observable wavelength regions with HERMES.

\section{The GALAH Survey Sample and Synergies}

The GALAH Survey to commence in 2013 will be a large observing program on the AAT. The survey will target approximately a million stars down to a V magnitude of $\sim 14$. Each target will be observed with HERMES to obtain high resolution spectra with a signal-to-noise ratio of over 100 per resolution element in each of the HERMES channels. Of the GALAH catalogue, 
thin disk stars provide about $78 \%$ of the sample. About $17 \%$ of the stars would be from the Galactic thick disk, and the remaining 5\% from the Galactic halo. The survey will observe Disk dwarf stars out to $\sim 1 \mathrm{kpc}$, the Disk giant stars to about $5 \mathrm{kpc}$, and the Halo giants to about 15 kpc.

The high resolution spectra from the four HERMES wavelength bands contain spectral lines of a large range of chemical elements, that includes light ( $\mathrm{Li})$, odd-z $(\mathrm{Na}, \mathrm{Al})$, alpha $(\mathrm{O}$, $\mathrm{Mg}, \mathrm{Si}, \mathrm{Ca}, \mathrm{Ti}$ ), Fe-peak (Cr, Mn, Fe, Co, Ni, Zn), light s-process (Y, Zr), heavy s-process (Ba, $\mathrm{La})$ and r-process $(\mathrm{Eu})$ elements. An automated abundance pipeline will use spectral synthesis to derive the elemental abundances with a relative accuracy of $0.05-0.1$ dex. Final data products of the survey will include precise abundances of well over 15 different chemical elements, accurate stellar parameters as well as high accuracy radial velocities per individual star for the entire million star catalogue.

The GALAH survey has strong synergies with many upcoming surveys, most notably with the SkyMapper Southern Sky survey and the European Space Agency's GAIA mission. SkyMapper will provide photometry in $\mathrm{u}, \mathrm{v}, \mathrm{g}, \mathrm{r}, \mathrm{i}$ and $\mathrm{z}$ filters and GAIA will measure precise distances and tangential motions for the GALAH survey stars. The combination of GALAH abundances, stellar parameters and radial velocities with SkyMapper photometry and GAIA astrometry will provide a truly extraordinary legacy for unraveling the history of the Galaxy.

\section{References}

[1] K. C. Freeman \& J. Bland-Hawthorn, 2002, ARAA, 40, 487

[2] S. Barden et al., 2010, SPIE, 7735, 9-19 [DOI:10.1117/12.856103] 